\title{
Utilizing Correlative Microscopy for the Analysis of Native Tissues and the Development of Novel Tissue Constructs for Regenerative Therapies
}

\author{
Ian Robinson ${ }^{1}$, James Miess ${ }^{1}$, Nikolai Sopko $\mathrm{MD}^{2}$ and Nicolas Baetz ${ }^{1^{*}}$ \\ 1. Department of Research and Development, Division of Cell and Tissue Imaging, PolarityTE, Salt \\ Lake City, UT USA. \\ 2. Department of Research and Development, PolarityTE, Salt Lake City, UT USA. \\ * Corresponding author: nbaetz@polarityte.com
}

Skin is the largest organ that includes multiple layers with important functions such as an environmental and antimicrobial barrier, thermal regulation, and fat storage. Bone is similarly important and serves and provides the structure support for containing all other organs and tissues. We explore the possibility of autologous homologous constructs to neo-generate a variety of fully functional tissue types to treat various clinical indications. Examining the ultrastructure and function of native tissue is crucial to produce a method to regenerate tissue similar in architecture and function to native tissue. To achieve this aim, we utilized correlative microscopy to analyze native, conventionally treated, naturally healed, and autologous homologous skin construct (AHSC) and autologous homologous bone construct (AHBC) treated tissues.

Designing novel regenerative tissue constructs requires examination of the necessary components of native tissues and their cellular niches to facilitate regeneration of skin/bone indistinguishable functionally and structurally from developmentally native tissue [1]. Using a variety of microscopy techniques including environmental scanning electron microscopy (ESEM), energy dispersive spectroscopy (EDS), second harmonic imaging (SHG), third harmonic imaging (THG), fluorescence lifetime imaging (FLIM), confocal, histology, immunohistochemistry (IHC), atomic force microscopy (AFM), and digital single lens reflex (DSLR) - tissues are imaged to determine correlative ultrastructural and cellular features within native and treated tissues [2]. Cross comparison of various modalities provides content specific data with clinical and translational applicability [3]. Donor native tissue specimens were obtained and interrogated through the various imaging modalities before and after our processing methods at an FDA-registered manufacturing facility to fully characterize their inherent properties. Whole mount imaging was completed using ESEM, SHG, THG, DSLR, EDS and confocal microscopes on excised formalin fixed tissues. Contrast media and clearing approaches were used to improve structural contrast and identify and characterize cellular populations. Native skin and bone and their respective constructs were paraffin embedded and sectioned in preparation for histology. Downstream immunological staining, ESEM, SHG, and AFM were performed to further elucidate the microarchitecture features and content of the treated and control specimen. The imaging results were correlated with in vitro and in vivo study outcomes to identify which processing formulations best recapitulated the properties of native skin and bone. Our methodology demonstrates that multimodal correlative microscopy-based investigations can be effectively applied to optimize regenerative product development.

\section{References:}

[1] M Georgiadis, R Müller and P Schneider, (2016). Journal of the Royal Society Interface 13 (2016), p. 119. https://doi.org/10.1098/rsif.2016.0088

[2] L Mostaço-Guidolin, NL Rosin and TL Hackett, International Journal of Molecular Sciences (2017). https://doi.org/10.3390/ijms18081772 
[3] R Cicchi et al., Journal of Biophotonics (2010). https://doi.org/10.1002/jbio.200910062

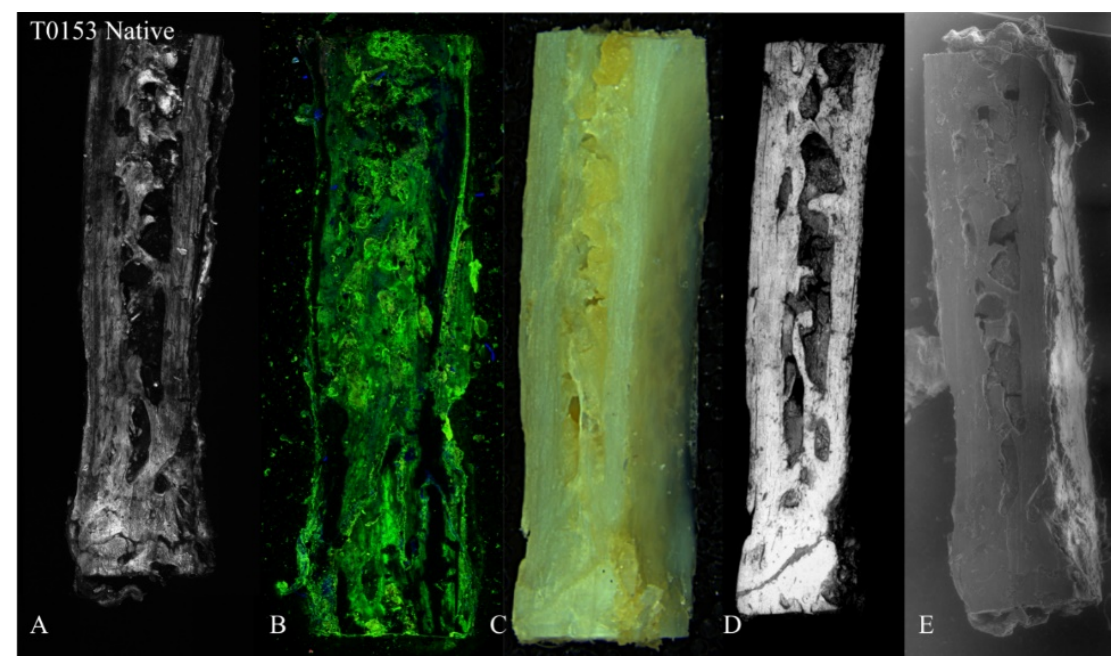

Figure 1. Native bone, (A) SHG (B) Confocal (C) Photo (D) ESEM backscatter detector (E) C2DX

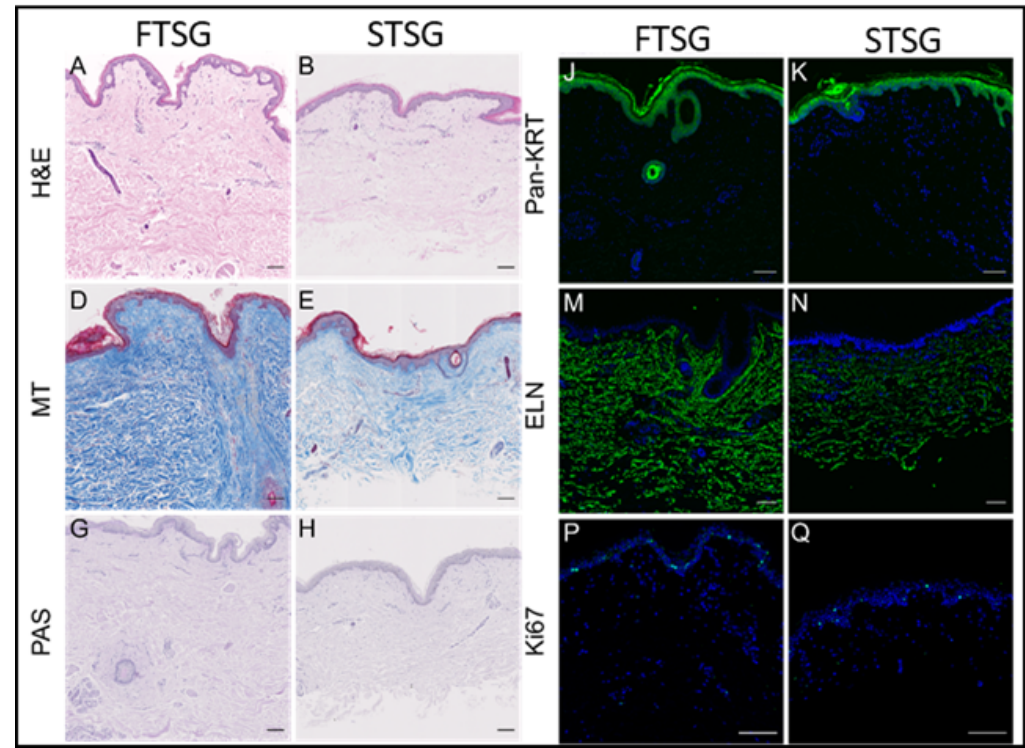

Figure 2. Histological and IHC analysis of conventional wound therapies - full-thickness skin graft (FTSG) tissue vs split-thickness skin graft (STSG). Scale bars $=100 \mu \mathrm{m}$. Full-thickness skin grafts (FTSG), split-thickness skin grafts (STSG) and processed skin tissue (AHSC) derived from the abdomen of a human donor were subjected to hematoxylin and eosin (H\&E, A-C), Masson's trichrome (MT, D-F) and periodic acid Schiff (PAS, G-I) histological staining and immunohistochemical (IHC) staining for pan-Cytokeratin (green, Pan-KRT, J-L), Elastin (green, ELN, M-O) and Ki67 (green, Ki67, P-R). IHC slides were counterstained with DAPI (blue). 